\title{
Mixed Boundary Value Problem on Hypersurfaces
}

\author{
R. DuDuchava, M. Tsaava, and T. Tsutsunava \\ A. Razmadze Mathematical Institute, Tbilisi State University, Tamarashvili Street 6, 0177 Tbilisi, Georgia
}

Correspondence should be addressed to M. Tsaava; m.caava@yahoo.com

Received 22 February 2014; Revised 31 May 2014; Accepted 2 June 2014; Published 17 August 2014

Academic Editor: Ioannis G. Stratis

Copyright (C) 2014 R. DuDuchava et al. This is an open access article distributed under the Creative Commons Attribution License, which permits unrestricted use, distribution, and reproduction in any medium, provided the original work is properly cited.

\begin{abstract}
The purpose of the present paper is to investigate the mixed Dirichlet-Neumann boundary value problems for the anisotropic Laplace-Beltrami equation $\operatorname{div}_{\mathscr{C}}\left(A \nabla_{\mathscr{C}} \varphi\right)=f$ on a smooth hypersurface $\mathscr{C}$ with the boundary $\Gamma=\partial \mathscr{C}$ in $\mathbb{R}^{n} . A(x)$ is an $n \times n$ bounded measurable positive definite matrix function. The boundary is decomposed into two nonintersecting connected parts $\Gamma=\Gamma_{D} \cup \Gamma_{N}$ and on $\Gamma_{D}$ the Dirichlet boundary conditions are prescribed, while on $\Gamma_{N}$ the Neumann conditions. The unique solvability of the mixed BVP is proved, based upon the Green formulae and Lax-Milgram Lemma. Further, the existence of the fundamental solution to $\operatorname{div}_{\mathcal{S}}\left(A \nabla_{\mathcal{S}}\right)$ is proved, which is interpreted as the invertibility of this operator in the setting $\mathbb{U}_{p, \#}^{s}(\mathcal{S}) \rightarrow \mathbb{U}_{p, \#}^{s-2}(\mathcal{S})$, where $\mathbb{U}_{p, \#}^{s}(\mathcal{S})$ is a subspace of the Bessel potential space and consists of functions with mean value zero.
\end{abstract}

\section{Introduction}

Let $\mathscr{C} \subset \mathcal{S}$ be a smooth subsurface of a closed hypersurface $\mathcal{S}$ in the Euclidean space $\mathbb{R}^{n}$ (see Section 2 for details) and let $\Gamma=\partial \mathscr{C} \neq \emptyset$ be its smooth boundary $\partial \mathscr{C}=\Gamma$. Let $\mathscr{D}_{j}:=$ $\partial_{j}-v_{j} \partial_{v}, j=1, \ldots, n$, be Günter's tangential derivatives, and let $\Delta_{\mathscr{C}}(t, \mathscr{D}):=\mathscr{D}_{1}^{2}+\cdots+\mathscr{D}_{n}^{2}$ be the Laplace-Beltrami operator restricted to the hypersurface $\mathscr{C}$ (see [1-3] and Section 2 below for details).

In [3], the boundary value problem for the LaplaceBeltrami equation with the Dirichlet boundary condition

$$
\begin{gathered}
\left(\Delta_{\mathscr{C}}(t, \mathscr{D}) u\right)(t)=f(t), \quad t \in \mathscr{C}, \\
u^{+}(s)=g(s), \quad \text { on } \Gamma
\end{gathered}
$$

and with the Neumann boundary condition

$$
\begin{gathered}
\left(\Delta_{\mathscr{C}}(t, \mathscr{D}) u\right)(t)=f(t), \quad t \in \mathscr{C}, \\
\left(\mathscr{D}_{\nu_{\Gamma}} u\right)^{+}(s)=h(s), \quad \text { on } \Gamma
\end{gathered}
$$

were considered where $\nu_{\Gamma}:=\left(\nu_{\Gamma, 1}, \ldots, \nu_{\Gamma, n}\right)^{\top}$ is the unit normal vector field to the boundary $\Gamma$ and tangent to the hypersurface $\mathscr{C}$ and $\varphi^{+}$denotes the trace on the boundary. The derivative

$$
\mathscr{D}_{\gamma_{\Gamma}}:=\sum_{k=1}^{n} \nu_{\Gamma, k} \mathscr{D}_{k}, \quad s \in \Gamma,
$$

is tangent to the hypersurface $\mathscr{C}$ and normal with respect to the boundary $\Gamma$.

The BVPs (1) and (2) were investigated in [3] in the following classical weak setting:

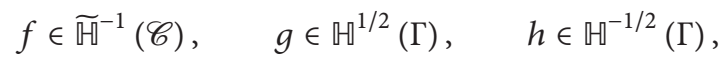

and also in nonclassical weak setting:

$$
\begin{array}{r}
f \in \widetilde{\mathbb{T}}_{p}^{s-2}(\mathscr{C}), \quad g \in \mathbb{W}_{p}^{s-1 / p}(\Gamma), \quad h \in \mathbb{W}_{p}^{s-1-1 / p}(\Gamma), \\
1<p<\infty, \quad s>\frac{1}{p},
\end{array}
$$

and the following was proved.

Theorem 1. The Dirichlet problems (1), (4) and (1), (5) have a unique solution.

For the solvability of the Neumann problems (2), (4) and (2), (5), the necessary and sufficient compatibility condition

$$
(f, 1)_{\mathscr{C}}-(h, 1)_{\Gamma}=0
$$


should be fulfilled, which guarantees the existence and the uniqueness of solution.

If $f$ and $h$ are regular integrable functions, the compatibility condition (6) acquires the form

$$
\int_{\mathscr{C}} f(y) d \sigma-\oint_{\Gamma} h(s) d s=0 .
$$

In Remarks 15 and 16, it is shown that the unique solvability of the Dirichlet BVP (1), (4) and the Neumenn BVP (2), (4) in the classical formulation follows from the LaxMilgram Lemma.

The investigation in [3] is based on the technique of Günter's derivatives developed in the preprint of Duduchava from 2002 and later in the paper of Duduchava et al. [2] and applies potential method. Similar problems, for $p=2$, by different technique were investigated earlier in the paper of Mitrea and Taylor [4].

The purpose of the present paper is to investigate the boundary value problems for the anisotropic Laplace equation with mixed boundary conditions:

$$
\begin{gathered}
\operatorname{div}_{\mathscr{C}}\left(A \nabla_{\mathscr{C}} u\right)(t)=f(t), \quad t \in \mathscr{C}, \\
u^{+}(s)=g(s), \quad \text { on } \Gamma_{D}, \\
\left\langle\nu_{\Gamma}(s),\left(A \nabla_{\mathscr{C}} u\right)^{+}(s)\right\rangle=h(s), \quad \text { on } \Gamma_{N},
\end{gathered}
$$

where $\partial \mathscr{C}=\Gamma=\Gamma_{D} \cup \Gamma_{N}$ is a decomposition of the boundary into two connected parts, $A=\left\{a_{i j}\right\}$ is an $n \times n$ strictly positive definite matrix, and

$$
\langle A(x) \xi, \xi\rangle \geqslant C\|\xi\|^{2}, \quad \xi \in \mathbb{R}^{n},
$$

for all $x \in \mathscr{C}$. We consider the BVP (8) in the weak classical setting (4).

The nonclassical weak setting (5) will be considered in a forthcoming paper.

Remark 2. As shown in [14], page 196, condition (4) does not ensure the uniqueness of solutions to the BVPs (1), (2) and (8). The right hand side $\mathrm{f}$ needs additional constraint that it belongs to the subspace $\widetilde{\mathbb{H}}_{0}^{-1}(\Omega) \subset \widetilde{\mathbb{M}}^{-1}(\Omega)$ which is the orthogonal complement to the subspace $\widetilde{\mathbb{M}}^{-1}(\Gamma)$ of those distributions from $\widetilde{\mathbb{U}}^{-1}(\Omega)$ which are supported on the boundary $\Gamma=\partial \Omega$ of the domain only.

For the non-classical setting (5) we schould impose a similar constraint $f \in \widetilde{\mathbb{M}}_{p, 0}^{-1}(\Omega) \subset \widetilde{\mathbb{H}}_{p}^{-1}(\Omega)$, which is defined as in the case $p=2$.

For the classical setting (4), we apply the Lax-Milgram Lemma and prove unique solvability of the problem rather easily, while (5) in the nonclassical investigation relies again on the potential method.

Mixed BVPs for the Laplace equation in domains were investigated by Lax-Milgram Lemma by many authors (see, e.g., the recent lecture notes online [5]).

BVPs on hypersurfaces arise in a variety of situations and have many practical applications. See, for example,
[6, Section 7.2] for the heat conduction by surfaces, [7, Section 10] for the equations of surface flow, $[8,9]$ for the vacuum Einstein equations describing gravitational fields, and [10] for the Navier-Stokes equations on spherical domains, as well as the references therein.

A hypersurface $\mathcal{S}$ in $\mathbb{R}^{n}$ has the natural structure of an $(n-1)$-dimensional Riemannian manifold and the aforementioned PDEs are not the immediate analogues of the ones corresponding to the flat, Euclidean case, since they have to take into consideration geometric characteristics of $\mathcal{S}$ such as curvature. Inherently, these PDEs are originally written in local coordinates, intrinsic to the manifold structure of $\mathcal{S}$.

Another problem considered in the present paper is the existence of a fundamental solution for the Laplace-Beltrami operator. An essential difference between differential operators on hypersurfaces and the Euclidean space $\mathbb{R}^{n}$ lies in the existence of fundamental solution: in $\mathbb{R}^{n}$ fundamental solution exists for all partial differential operators with constant coefficients if it is not trivially zero. On a hypersurface even Laplace-Beltrami operator does not have a fundamental solution because it has a nontrivial kernel, constants, in all Bessel potential spaces. Therefore we consider LaplaceBeltrami operator in Hilbert spaces with detached constants $\mathbb{W}_{p, \#}^{s}(\mathcal{S}) \rightarrow \mathbb{W}_{p, \#}^{s-2}(\mathcal{S})$, for all $1<p<\infty, s \in \mathbb{R}$, and prove that it is an invertible operator. Another description of the space $\mathbb{W}_{p, \#}^{s}(\mathcal{S})$ is that it consists of all functions $\varphi \in$ $\mathbb{W}_{p}^{s}(\mathcal{S})$ (distributions if $s<0$, which have the zero mean value, $\left.(\varphi, 1)_{\mathcal{S}}=0\right)$. The established invertibility implies the existence of the certain fundamental solution, which can be used to define the volume (Newtonian), single layer, and double layer potentials.

The structure of the paper is as follows. In Section 2, we expose all necessary definitions and some auxiliary material, partly new ones. Here the invertibility of the Laplace-Beltrami operator in the setting $\mathbb{W}_{p, \#}^{s}(\mathcal{S}) \rightarrow \mathbb{W}_{p, \#}^{s-2}(\mathcal{S})$ is proved. In Section 3, using the Lax-Milgram Lemma, it is proved that the basic mixed BVP (8) has a unique solution in the weak classical setting (4).

\section{Auxiliary Material}

We commence with definitions of a hypersurface. There exist other equivalent definitions but these are the most convenient for us. Equivalence of these definitions and some other properties of hypersurfaces are exposed, for example, in $[3,11]$.

Definition 3. A subset $\mathcal{S} \subset \mathbb{R}^{n}$ of the Euclidean space is called a hypersurface if it has a covering $\mathcal{S}=\bigcup_{j=1}^{M} \mathcal{S}_{j}$ and coordinate mappings

$$
\begin{array}{r}
\Theta_{j}: \omega_{j} \longrightarrow \mathcal{S}_{j}:=\Theta_{j}\left(\omega_{j}\right) \subset \mathbb{R}^{n}, \\
\omega_{j} \subset \mathbb{R}^{n-1}, \quad j=1, \ldots, M,
\end{array}
$$

such that the corresponding differentials

$$
D \Theta_{j}(p):=\operatorname{matr}\left[\partial_{1} \Theta_{j}(p), \ldots, \partial_{n-1} \Theta_{j}(p)\right]
$$


have the full rank

$$
\begin{array}{r}
\operatorname{rank} D \Theta_{j}(p)=n-1, \\
\forall p \in Y_{j}, \quad k=1, \ldots, n, \quad j=1, \ldots, M ;
\end{array}
$$

that is, all points of $\omega_{j}$ are regular for $\Theta_{j}$ for all $j=1, \ldots, M$.

Such a mapping is called an immersion as well.

Here and in what follows $\operatorname{matr}\left[x_{1}, \ldots, x_{k}\right]$ refers to the matrix with the listed vectors $x_{1}, \ldots, x_{k}$ as columns.

A hypersurface is called smooth if the corresponding coordinate diffeomorphisms $\Theta_{j}$ in $(10)$ are smooth $\left(C^{\infty}\right.$ smooth). Similarly is defined a $\mu$-smooth hypersurface.

The next definition of a hypersurface is implicit.

Definition 4. Let $k \geqslant 1$ and $\omega \subset \mathbb{R}^{n}$ be a compact domain. An implicit $C^{k}$-smooth hypersurface in $\mathbb{R}^{n}$ is defined as the set

$$
\mathcal{S}=\left\{\mathscr{X} \in \omega: \Psi_{\mathcal{S}}(\mathscr{X})=0\right\},
$$

where $\Psi_{\mathcal{\delta}}: \omega \rightarrow \mathbb{R}$ is a $C^{k}$-mapping, which is regular $\nabla \Psi(\mathscr{X}) \neq 0$

Stoke's derivatives are concrete examples of tangential operators

$$
\mathscr{M}_{\mathcal{S}}:=\left[\mathscr{M}_{j k}\right]_{n \times n}, \quad \mathscr{M}_{j k}:=v_{j} \partial_{k}-v_{k} \partial_{j}=\partial \operatorname{rtial}_{m_{j, k}} .
$$

Corollary 5 (Green's formula; cf. [1]). Let $\Omega \subset \mathbb{R}^{n}$ be a domain with Lipschitz boundary. For the Laplace operator

$$
\Delta:=\partial_{1}^{2}+\cdots+\partial_{n}^{2}
$$

and functions $\varphi, \psi \in \mathbb{W}_{2}^{1}(\Omega), \Delta \psi \in \widetilde{\mathbb{W}}^{1}(\Omega)$, the following I and II Green formulae are valid:

$$
\begin{aligned}
& \int_{\Omega}(\Delta \psi)(y) \varphi(y) d y \\
&= \oint_{\partial \Omega}\left(\partial_{\nu} \psi\right)(\tau) \varphi(\tau) d \sigma \\
&-\sum_{j=1}^{n} \int_{\Omega}\left(\partial_{j} \psi\right)(y)\left(\partial_{j} \varphi\right)(y) d y \\
& \int_{\Omega}(\Delta \psi)(y) \varphi(y) d y \\
&=\int_{\Omega} \psi(y)(\Delta \varphi)(y) d y \\
& \quad+\oint_{\partial \Omega}\left[\left(\partial_{\nu} \psi\right)(\tau) \varphi(\tau)+\psi(\tau)\left(\partial_{\nu} \varphi\right)(\tau)\right] d \sigma .
\end{aligned}
$$

Integrals in (16) are understood in the sense of duality between spaces $\widetilde{\mathbb{W}}^{-1}(\Omega)$ and $\mathbb{W}^{1}(\Omega), \mathbb{H}^{-1 / 2}(\partial \Omega)$ and $\mathbb{M}^{1 / 2}(\partial \Omega)$, and so forth.

Let $\mathcal{S}$ be a closed hypersurface in $\mathbb{R}^{n}$ and let $\mathscr{C}$ be a smooth subsurface of $\mathcal{S}$, given by an immersion

$$
\Theta: \omega \longrightarrow \mathscr{C}, \quad \omega \subset \mathbb{R}^{n-1}
$$

with a boundary $\Gamma=\partial \mathscr{C}$, given by another immersion

$$
\Theta_{\Gamma}: \omega \longrightarrow \Gamma:=\partial \mathscr{C}, \quad \omega \subset \mathbb{R}^{n-2},
$$

and let $\boldsymbol{\nu}(\mathscr{X})$ be the outer unit normal vector field to $\mathscr{C}$ and let $\mathcal{N}(x)$ denote an extended unit field in a neighborhood $\omega_{\mathscr{C}}$ of $\mathscr{C} . \nu_{\Gamma}(t)$ is the outer normal vector field to the boundary $\Gamma$, which is tangential to $\mathscr{C}$.

A curve on a smooth surface $\mathscr{C}$ is a mapping

$$
\gamma: \mathscr{I} \longmapsto \mathscr{C}, \quad \mathscr{I}:=(a, b] \subset \mathbb{R},
$$

of a line interval $\mathscr{I}$ to $\mathscr{C}$.

A vector field on a domain $\Omega$ in $\mathbb{R}^{n}$ is a mapping

$$
\mathbf{U}: \Omega \longrightarrow \mathbb{R}^{n}, \quad \mathbf{U}(x)=\sum_{j=1}^{n} U_{j}(x) \mathbf{e}^{j},
$$

where $U^{j} \in C_{0}^{\infty}(\Omega)$ and $\mathbf{e}^{j}$ is the element of the natural Cartesian basis in $\mathbb{R}^{n}$ :

$$
e^{1}:=(1,0, \ldots, 0), \ldots, e^{n}:=(0, \ldots, 0,1),
$$

in the Euclidean space $\mathbb{R}^{n}$.

By $\mathscr{V}(\Omega)$ we denote the set of all smooth vector fields on $\Omega$.

A vector field $\mathbf{U} \in \mathscr{V}(\Omega)$ defines the first order differential operator

$$
\begin{aligned}
& \mathbf{U} f(x)=\operatorname{drtial}_{\mathbf{U}} f(x):=\lim _{h \rightarrow 0} \frac{f\left(\mathscr{F}_{\mathbf{U}}^{h}(x)\right)-f(x)}{h} \\
& =\left.\frac{d}{d t} f\left(\mathscr{F}_{\mathrm{U}}^{t}(x)\right)\right|_{t=0} \text {, }
\end{aligned}
$$

where $\mathscr{F}_{\mathrm{U}}^{t}(x)$ is the orbit of the vector field $U$.

Let

$$
P(D) u=\sum_{j=1}^{n} a_{j} \partial_{j} u+b u, \quad a_{j}, b \in C^{1}\left(\mathbb{R}^{m \times m}\right),
$$

be a first order differential operator with real valued (variable) matrix coefficients, acting on vector-valued functions in $\mathbb{R}^{n}$, and its principal symbol is given by the matrix-valued function

$$
\sigma(P ; \xi):=\sum_{j=1}^{n} a_{j} \xi_{j}, \quad \xi=\left\{\xi_{j}\right\}_{j=1}^{n} \in \mathbb{R}^{n}
$$

To distinguish an open and a closed hypersurface, we use the notation $\mathcal{S}$ for a closed hypersurface without the boundary $\partial$ rtial $\mathcal{S}=\emptyset$ (we remind the reader that the notation $\mathscr{C}$ is reserved for an open hypersurface with the boundary $\Gamma:=\partial \operatorname{rtial} \mathcal{S})$.

Definition 6. We say that $P$ is a tangential operator to the hypersurface $\mathcal{S}$, with unit normal $\boldsymbol{v}$, if

$$
\sigma(P ; \boldsymbol{v})=0 \text { on the hypersurface } \mathcal{S} \text {. }
$$


Lemma 7. Let $P$ be, as in (23), a first order differential operator with $C^{1}$-smooth coefficients. $P$ is tangential if and only if the adjoint $P^{*}$ operator is tangential.

If $P$ is tangential to $\mathcal{S}$ and $P$ is defined in a neighborhood of $\mathcal{S}$, then

$$
\left.(P \varphi)\right|_{\mathcal{\delta}}=P\left(\left.\varphi\right|_{\delta}\right),
$$

for every $C^{1}$ function $\varphi$ defined in a neighborhood of $\mathcal{S}$.

We continue with the definition of the surface divergence $\operatorname{div}_{\mathcal{S}}$, the surface gradient $\nabla_{\mathcal{S}}$, and the surface LaplaceBeltrami operator $\Delta_{\delta}$.

According to the classical differential geometry, the surface gradient $\nabla_{\mathcal{\delta}}$ of a function $f \in C^{1}(\mathcal{S})$ is defined by

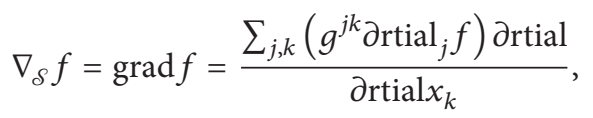

and the surface divergence of a smooth tangential vector field $\mathbf{V}$ is defined by

$$
\operatorname{div}_{\mathcal{S}} \mathbf{V}:=\sum_{k=1}^{n-1} V_{; j}^{j}, \quad V_{; k}^{j}:=\partial_{k} V^{j}+\sum_{m=1}^{n-1} \Gamma_{k m}^{j} V^{m},
$$

where $\Gamma_{k m}^{j}$ denotes the Christoffel symbols

$$
\Gamma_{k m}^{j}:=\frac{1}{2} \sum_{k=1}^{n-1} g^{j \ell}\left[\partial_{m} g_{k \ell}+\partial_{k} g_{m \ell}-\partial_{\ell} g_{k m}\right]=\Gamma_{m k}^{j}
$$

and $G:=\left[g_{j k}\right]$ is the covariant Riemann metric tensor, while $G^{-1}:=\left[g^{j k}\right]$ is the inverse to it-the contravariant Riemann tensor.

$\operatorname{div}_{\delta}$ is the negative dual to the surface gradient:

$$
\left\langle\operatorname{div}_{\mathcal{\delta}} \mathbf{V}, f\right\rangle:=-\left\langle\mathbf{V}, \nabla_{\delta} f\right\rangle, \quad \forall \mathbf{V} \in \mathscr{V}(\mathcal{S}), \forall f \in C^{1}(\mathcal{S}) .
$$

The Laplace-Beltrami operator $\Delta_{\mathcal{\delta}}$ on $\mathcal{S}$ is defined as the composition

$$
\Delta_{\mathcal{\delta}} \psi=\operatorname{div}_{\mathcal{\delta}} \nabla_{\mathcal{\delta}} \psi=-\nabla_{\mathcal{S}}^{*}\left(\nabla_{\mathcal{\delta}} \psi\right) .
$$

Theorem 8 (cf. [2]). For any function $\varphi \in C^{1}(\mathcal{S})$, one has

$$
\nabla_{\delta} \varphi=\left\{\mathscr{D}_{1} \varphi, \mathscr{D}_{2} \varphi, \ldots, \mathscr{D}_{n} \varphi\right\}^{\top} .
$$

Also, for a 1-smooth tangential vector field $\mathbf{V}=\sum_{j=1}^{n} V^{j} e_{j} \in$ $\mathscr{V}(\mathcal{S})$

$$
\operatorname{div}_{\mathcal{S}} \mathbf{V}=-\nabla_{\mathcal{S}}^{*} \mathbf{V}:=\sum_{j=1}^{n} \mathscr{D}_{j} V^{j} .
$$

The Laplace-Beltrami operator $\Delta_{\mathcal{\delta}}$ on $\mathcal{S}$ takes the form

$$
\Delta_{\mathcal{S}} \psi=\sum_{j=1}^{n} \mathscr{D}_{j}^{2} \psi=\sum_{j<k} \mathscr{M}_{j k}^{2} \psi=\frac{1}{2} \sum_{j, k=1}^{n} \mathscr{M}_{j k}^{2} \psi, \quad \forall \psi \in C^{2}(\mathcal{S}) .
$$

Corollary 9 (cf. [2]). Let $\delta$ be a smooth closed hypersurface. The homogeneous equation

$$
\Delta_{\mathcal{\delta}} \psi=0
$$

has only a constant solution in the space $\mathbb{W}^{1}(\mathcal{S})$.

Proof. Due to (31) and (35), we get

$$
0=\left(-\Delta_{\delta} \psi, \psi\right)=\left(\nabla_{\delta} \psi, \nabla_{\delta} \psi\right)=\left\|\nabla_{\delta} \psi \mid \mathbb{L}_{2}(\mathcal{S})\right\|,
$$

which gives $\nabla_{\delta} \psi=0$. But the trivial surface gradient means constant function $\psi=$ const (this is easy to ascertain by analysing the definition of Günter's derivatives; see, e.g., [3]).

Let $\mathscr{M}$ be a nontrivial mes $\mathscr{M} \neq \emptyset$, smooth closed or open hypersurface, $s \in \mathbb{R}$, and $1<p<\infty$. For the definitions of Bessel potential $\mathbb{T}_{p}^{s}(\mathscr{M})$ and Sobolev-Slobodeckii $\mathbb{W}_{p}^{s}(\mathscr{M})$ spaces for a closed smooth manifold $\mathscr{M}$, we refer to [12] (also see $[1,13,14])$. For $p=2$ the Sobolev-Slobodetski $\mathbb{W}_{2}^{s}(\mathscr{M})$ and Bessel potential $\mathbb{M}_{2}^{s}(\Gamma)$ spaces coincide (i.e., the norms are equivalent).

Let $\mathscr{C}$ be a subsurface of a smooth closed surface $\mathscr{M}, \mathscr{C} C$ $\mathscr{M}$, with the smooth boundary $\Gamma:=\partial \operatorname{rtial} \mathcal{S}$. The space $\widetilde{\mathbb{U}}_{p}^{s}(\mathscr{C})$ is defined as the subspace of those functions $\varphi \in \mathbb{T}_{p}^{s}(\mathscr{M})$, which are supported in the closure of the subsurface, supp $\varphi \subset$ $\overline{\mathscr{C}}$, whereas $\mathbb{T}_{p}^{s}(\mathscr{C})$ denotes the quotient space $\mathbb{U}_{p}^{s}(\mathscr{C})=$ $\mathbb{M}_{p}^{s}(\mathscr{M}) / \widetilde{\mathbb{U}}_{p}^{s}\left(\mathscr{C}^{c}\right)$, and $\mathscr{C}^{c}:=\mathscr{M} \backslash \overline{\mathscr{C}}$ is the complementary subsurface to $\mathscr{C}$. The space $\mathbb{T}_{p}^{s}(\mathscr{C})$ can be identified with the space of distributions $\varphi$ on $\mathscr{C}$ which have an extension to a distribution $\ell \varphi \in \mathbb{T}_{p}^{s}(\mathscr{M})$. Therefore, $r_{\mathscr{C}} \mathbb{W}_{p}^{s}(\mathscr{M})=$ $\mathbb{\mathbb { V } _ { p } ^ { s }}(\mathscr{C})$, where $r_{\mathscr{C}}$ denotes the restriction operator of functions (distributions) from the surface $\mathscr{M}$ to the subsurface $\mathbb{R}^{n}$.

The spaces $\widetilde{\mathbb{W}}_{p}^{s}(\mathscr{C})$ and $\mathbb{W}_{p}^{s}(\mathscr{C})$ are defined similarly (see $[12]$ and also $[1,13,14])$.

By $\mathbb{X}_{p}^{s}(\mathscr{M})$ we denote one of the spaces: $\mathbb{W}_{p}^{s}(\mathscr{M})$ and Sobolev-Slobodecki $\mathbb{W}_{p}^{s}(\mathscr{M})$ (if $\mathscr{M}$ is closed or open), and by $\widetilde{\mathbb{X}}_{p}^{s}(\mathscr{M})$ denote one of the spaces: $\widetilde{\mathbb{T}}_{p}^{s}(\mathscr{M})$ and $\widetilde{\mathbb{W}}_{p}^{s}(\mathscr{M})$ (if $\mathscr{M}$ is open). Consider the space

$$
\mathbb{X}_{p, \#}^{s}(\mathscr{M}):=\left\{\varphi \in \mathbb{X}_{2}^{s}(\mathscr{M}):(\varphi, 1)=0\right\} .
$$

It is obvious that $\mathbb{X}_{p, \#}^{s}(\mathscr{M})$ does not contain constants: if $c_{0}=$ const $\in \mathbb{X}_{p, \#}^{s}(\mathscr{M})$, then

$$
0=\left(c_{0}, 1\right)=c_{0}(1,1)=c_{0} \operatorname{mes} \mathscr{M}
$$

and $c_{0}=0$. Moreover, $\mathbb{X}_{p}^{s}(\mathscr{M})$ decomposes into the direct sum

$$
\mathbb{X}_{p}^{s}(\mathscr{M})=\mathbb{X}_{p, \#}^{s}(\mathscr{M})+\{\text { const }\}
$$

and the dual (adjoint) space is

$$
\left(\mathbb{X}_{p, \#}^{s}(\mathscr{M})\right)^{*}=\mathbb{X}_{p^{\prime}, \#}^{-s}(\mathscr{M}), \quad p^{\prime}:=\frac{p}{p-1} .
$$


In fact, the decomposition (39) follows from the representation

$$
\varphi=\varphi_{0}+\varphi_{\text {aver }}, \quad \varphi_{0} \in \mathbb{X}_{p, \#}^{s}(\mathscr{M}), \varphi_{\text {aver }}:=\frac{1}{\operatorname{mes} \mathscr{M}}(\varphi, 1)
$$

of arbitrary function $\varphi \in \mathbb{X}_{p}^{s}(\mathscr{M})$, because the average of the difference of a function and its average is zero: $\left(\varphi_{0}\right)_{\text {aver }}=$ $\left(\varphi-\varphi_{\text {aver }}\right)_{\text {aver }}=0$.

Since the Sobolev space $\mathbb{W}_{p, \#}^{m}(\mathscr{M})$ with integer smoothness parameter $m=1,2, \ldots$ does not contain constants, due to Corollary 9 the equivalent norm in this space can also be defined as follows:

$$
\left\|\varphi\left|\mathbb{W}_{p, \#}^{m}(\mathscr{M})\left\|_{0}:=\sum_{1 \leqslant|\alpha| \leqslant m}\right\| \mathscr{D}^{\alpha} \varphi\right| \mathbb{L}_{p}(\mathscr{M})\right\|
$$

In particular, in the space $\mathbb{W}_{p, \#}^{1}(\mathscr{M})$ the equivalent norm is

$$
\left\|\varphi\left|\mathbb{W}_{p, \#}^{1}(\mathscr{M})\left\|_{0}:=\right\| \nabla_{\delta} \varphi\right| \mathbb{L}_{p}(\mathscr{M})\right\| \text {. }
$$

The description (40) of the dual space follows from the fact that the dual space to $\mathbb{X}_{p}^{s}(\mathscr{M})$ is $\mathbb{X}_{p^{\prime}}^{-s}(\mathscr{M})$ (see [12]) and, therefore, due to the decomposition (39) and Hahn-Banach theorem the dual space to $\mathbb{X}_{p, \#}^{s}(\mathscr{M})$ should be embedded into $\mathbb{X}_{p^{\prime}}^{-s}(\mathscr{M})$. The only functional from $\mathbb{X}_{p^{\prime}}^{-s}(\mathscr{M})$ that vanishes on the entire space $\mathbb{X}_{p, \#}^{s}(\mathscr{M})$ is constant $1 \in \mathbb{X}_{p^{\prime}}^{-s}(\mathscr{M})$ (see definition (37)). After detaching this functional the remainder coincides, due to (39), with the space $\mathbb{X}_{p^{\prime}, \#}^{-s}(\mathscr{M})$, which is the dual to $\mathbb{X}_{p, \#}^{s}(\mathscr{M})$.

Theorem 10. Let $\mathcal{S}$ be $\ell$-smooth $\ell=1,2, \ldots, 1<p<\infty$ and $|s| \leqslant \ell$. Let $\mathbb{X}_{p}^{s}(\mathcal{S})$ be the same as in (37)-(40).

Let $\mathscr{H} \in C^{\ell-1}\left(\mathbb{R}^{n}\right)$ have one of the following properties:

(i) $\mathscr{H}$ has a nonnegative real part $\operatorname{Re} \mathscr{H}(t) \geqslant 0$, for all $t \in \mathcal{S}$, and mes supp $\operatorname{Re} \mathscr{H} \neq 0$;

(ii) $\mathscr{H}$ has a constant complex part $\operatorname{Im} \mathscr{H}(t)=$ const $\neq 0$;

(iii) $\operatorname{Re} \mathscr{H}=0$, mes supp Im $\mathscr{H} \neq 0$, and the complex part $\operatorname{Im} \mathscr{H}$ does not change the sign: either $\operatorname{Im} \mathscr{H}(t) \geqslant 0$, for all $t \in \mathcal{S}$, or $\operatorname{Im} \mathscr{H}(t) \leqslant 0$, for all $t \in \mathcal{S}$.

The perturbed operator

$$
\operatorname{div}_{\mathcal{S}} A \nabla_{\mathcal{S}}-\mathscr{H} I: \mathbb{X}_{p}^{s+1}(\mathcal{S}) \longrightarrow \mathbb{X}_{p}^{s-1}(\mathcal{S})
$$

is invertible, which can be interpreted as the existence of the fundamental solution to $\operatorname{div}_{\mathcal{S}} A \nabla_{\mathcal{S}}-\mathscr{H} I$.

The operator $\operatorname{div}_{\mathcal{S}} A \nabla_{\mathcal{S}}$ itself is invertible between the spaces with detached constants (see (37))

$$
\operatorname{div}_{\mathcal{S}} A \nabla_{\mathcal{S}}: \mathbb{X}_{p, \#}^{s+1}(\mathcal{S}) \longrightarrow \mathbb{X}_{p, \#}^{s-1}(\mathcal{S})
$$

And, therefore, $\operatorname{div}_{\delta} A \nabla_{\mathcal{S}}$ has the fundamental solution in the setting (45).
Proof. The first part of the theorem is proved in [3, Theorem 7.1] for the space setting $\mathbb{W}^{1}(\mathcal{S}) \rightarrow \mathbb{W}^{-1}(\mathcal{S})$ only. Therefore, we will prove it here in full generality.

First of all, note that the operator (44) is bounded and elliptic, as an elliptic operator on the closed hypersurface $\operatorname{div}_{\mathcal{S}} A \nabla_{\mathcal{S}}-\mathscr{H} I$ in (44) is Fredholm, for all $s \in \mathbb{R}$ and $1<$ $p<\infty$ (it has a parametrix if $\mathcal{S}$ is infinitely smooth; see $[13,15,16])$. On the other hand,

$$
\begin{aligned}
(- & \left.\left(\operatorname{div}_{\mathcal{\delta}} A \nabla_{\mathcal{\delta}}-\mathscr{H}\right) \varphi, \varphi\right)_{L_{2}(\delta)} \\
= & -\int_{\Gamma}\left\langle\left(\nu_{\Gamma}, A \nabla_{\mathcal{\delta}} \varphi\right)^{+}, \varphi^{+}\right\rangle d s \\
& +\left(A \nabla_{\mathcal{\delta}} \varphi, \nabla_{\mathcal{\delta}} \varphi\right)_{\mathcal{S}}+(\operatorname{Re} \mathscr{H} \varphi, \varphi)_{\mathcal{S}} \\
& +i(\operatorname{Im} \mathscr{H} \varphi, \varphi)_{\mathcal{S}} \quad \forall \varphi \in \mathbb{W}_{2}^{1}(\mathcal{S}) .
\end{aligned}
$$

Let us prove the uniqueness of the solution. For this, consider homogenous boundary conditions: $f=0,\left(\nu_{\Gamma}, A \nabla_{\mathcal{S}} \varphi\right)=$ 0 on $\Gamma_{N}$ and $\varphi^{+}=0$ on $\Gamma_{D}$. Then, $\left(\operatorname{div}_{\mathcal{S}} A \nabla_{\mathcal{S}}-\mathscr{H}\right) \varphi=0$ and $\int_{\Gamma}\left\langle\left(\nu_{\Gamma}, A \nabla_{\mathcal{S}} \varphi\right)^{+}, \varphi^{+}\right\rangle d s=0$, and finally we get

$$
\left(A \nabla_{\delta} \varphi, \nabla_{\delta} \varphi\right)_{\mathcal{S}}+(\operatorname{Re} \mathscr{H} \varphi, \varphi)_{\mathcal{S}}=0, \quad(\operatorname{Im} \mathscr{H} \varphi, \varphi)_{\mathcal{\delta}}=0 .
$$

Now let $\operatorname{Re} \mathscr{H}(t) \geqslant 0$, for all $t \in \mathcal{S}$, and mes supp $\operatorname{Re} \mathscr{H} \neq 0$ (case (i)). Then from the first equality in (47), it follows that

$$
\left(A \nabla_{\delta} \varphi, \nabla_{\delta} \varphi\right)_{\mathcal{S}}=0, \quad(\operatorname{Re} \mathscr{H} \varphi, \varphi)_{\mathcal{S}}=0 .
$$

The first equality $\left(A \nabla_{\delta} \varphi, \nabla_{\delta} \varphi\right)_{\mathcal{S}}=0$ yields $\varphi(t)=C=$ const and by inserting this in the second one we get

$$
0=(\operatorname{Re} \mathscr{H} \varphi, \varphi)_{\mathcal{S}}=C \int_{\mathcal{S}} \mathscr{H}(t) d \sigma,
$$

and the conclusion $\varphi(t)=C=$ const $=0$ is immediate.

If $M=\operatorname{Im} \mathscr{H}(t)=$ const $\neq 0$ (case (ii)), the same conclusion follows from the second equality in (47):

$$
0=(\operatorname{Im} \mathscr{H} \varphi, \varphi)_{\mathcal{S}}=M(\varphi, \varphi)_{\mathcal{S}}=M\left\|\varphi \mid L_{2}(\mathcal{S})\right\|^{2},
$$

and, again, $\varphi=0$.

If $\operatorname{Re} \mathscr{H}=0$, either $\operatorname{Im} \mathscr{H}(t) \geqslant 0$, for all $t \in \mathcal{S}$, or Im $\mathscr{H}(t) \leqslant 0$, for all $t \in \mathcal{S}$, and mes supp Im $\mathscr{H} \neq 0$ (case (iii)); from equality (46) it follows that

$$
\begin{aligned}
&(-\left.\left(\operatorname{div}_{\mathcal{S}} A \nabla_{\mathcal{S}}-\mathscr{H}\right) \varphi, \varphi\right)_{\mathcal{S}} \\
& \quad=\left(A \nabla_{\mathcal{S}} \varphi, \nabla_{\mathcal{S}} \varphi\right)_{\mathcal{S}}+i(\operatorname{Im} \mathscr{H} \varphi, \varphi)_{\mathcal{S}}=0
\end{aligned}
$$

and, consequently, if $\left(\operatorname{div}_{\mathcal{S}} A \nabla_{\mathcal{S}}-\mathscr{H}\right)_{\mathcal{\delta}} \varphi=0$ for $\mathbb{W}_{2}^{1}(\mathcal{S})$, then

$$
\left(A \nabla_{\delta} \varphi, \nabla_{\delta} \varphi\right)_{\mathcal{S}}=0, \quad(\operatorname{Im} \mathscr{H} \varphi, \varphi)_{\mathcal{S}}=0 .
$$

The conclusion $\varphi=$ const $=0$ follows as in the case (i).

Therefore, $\operatorname{Ker}\left(\operatorname{div}_{\mathcal{S}} A \nabla_{\mathcal{S}}-\mathscr{H} I\right)=\{0\}$. Since the operator is self-adjoint, the same is true for the dual operator Coker $\left(\operatorname{div}_{\mathcal{\delta}} A \nabla_{\mathcal{\delta}}-\mathscr{H} I\right)=\operatorname{Ker}\left(\operatorname{div}_{\mathcal{\delta}} A \nabla_{\mathcal{\delta}}-\right.$ $\mathscr{H} I)=\{0\}$ which, together with the Fredholm property of 
$\operatorname{div}_{\mathcal{S}} A \nabla_{\mathcal{S}}-\mathscr{H} I: \mathbb{W}^{1}(\mathcal{S}) \rightarrow \mathbb{W}^{-1}(\mathcal{S})$, yields the invertibility of this operator (of the operator in (44) for $s=0$ and $p=2$ ).

If $s \neq 0$ or $p \neq 2$, we proceed as follows. It is well known (see, e.g., [17]) that if an operator is Fredholm in the scale of Banach spaces (44), for all $s \in \mathbb{R}, 1<p<\infty$, and is invertible for only one pair of parameters $(p, s)=(2,0)$, it is invertible for all values of the parameters $s \in \mathbb{R}, 1<p<\infty$.

To prove the second assertion (see [3, Lemma 5.2] for a particular case), we note that the natural domain of the operator $\operatorname{div}_{\mathcal{S}} A \nabla_{\mathcal{S}}$ is $\mathbb{X}_{p, \#}^{s+1}(\mathcal{S})$.

To prove that the image of the operator $\operatorname{div}_{\mathcal{S}} A \nabla_{\mathcal{S}}$ coincides with the space $\mathbb{X}_{p, \#}^{s-1}(\mathcal{S})$, let $\psi_{0} \in \mathbb{X}_{p \text {,\# }}^{s-1}(\mathcal{S})$ be orthogonal to the image $\operatorname{Im} \operatorname{div}_{\mathcal{S}} A \nabla_{\mathcal{\delta}}$ of the operator. Then the equality

$$
0=\left(\operatorname{div}_{\mathcal{S}} A \nabla_{\mathcal{\delta}} \varphi, \psi_{0}\right)=\left(\varphi, \operatorname{div}_{\mathcal{\delta}} A \nabla_{\mathcal{S}} \psi_{0}\right)
$$

holds for all $\varphi \in \mathbb{X}_{p}^{s+1}(\mathcal{S})$. But then $\operatorname{div}_{\mathcal{S}} A \nabla_{\mathcal{\delta}} \psi_{0}=0$, which implies $\psi_{0}=$ const and, therefore, constants are only functions orthogonal to the image of the operator $\operatorname{Im} \operatorname{div}_{\mathcal{S}} A \nabla_{\mathcal{S}}$. This proves that the image $\operatorname{Im} \operatorname{div}_{\mathcal{S}} A \nabla_{\mathcal{S}}:=$ $\operatorname{div}_{\mathcal{S}} A \nabla_{\mathcal{S}} p$ coincides with the space $\mathbb{X}_{p, \#}^{s-1}(\mathcal{S})$.

Now, note that the operator $-\operatorname{div}_{\mathcal{S}} A \nabla_{\mathcal{S}}$ in the setting

$$
-\operatorname{div}_{\mathcal{S}} A \nabla_{\mathcal{S}}: \mathbb{W}_{2, \#}^{1}(\mathcal{S}) \longrightarrow \mathbb{W}_{2, \#}^{-1}(\mathcal{S})
$$

is positive definite (coercive). Indeed, due to (9), (31), and (43), we get

$$
\begin{aligned}
\left(-\operatorname{div}_{\mathcal{S}} A \nabla_{\delta} \varphi, \varphi\right) & =\left(A \nabla_{\mathcal{S}} \varphi, \nabla_{\mathcal{\delta}} \varphi\right) \\
\geq C\left\|\nabla_{\mathcal{S}} \varphi\left|\mathbb{L}_{2}(\mathcal{S})\left\|^{2}=C\right\| \varphi\right| \mathbb{W}_{2, \#}^{1}(\mathcal{S})\right\|^{2} & \forall \varphi \in \mathbb{W}_{2, \#}^{1}(\mathcal{S}) .
\end{aligned}
$$

Therefore, $\operatorname{div}_{\mathcal{S}} A \nabla_{\mathcal{\delta}}$ has the trivial kernel $\operatorname{Ker}\left(\operatorname{div}_{\mathcal{S}} A \nabla_{\mathcal{S}}\right)=$ $\{0\}$ in $\mathbb{W}_{2, \#}^{1}(\mathcal{S})$ and is normally solvable (has the closed image). Since $\operatorname{div}_{\mathcal{S}} A \nabla_{\mathcal{S}}$ is self-adjoint and the spaces $\mathbb{W}_{2, \#}^{1}(\mathcal{S})$ and $\mathbb{W}_{2, \#}^{-1}(\mathcal{S})$ are adjoint (see (40)), the adjoint operator to (54) is normally solvable as well and has a trivial kernel.

The proof is accomplished as in the first part of the theorem.

Corollary 11 (cf. [3]). For the operator $\operatorname{div}_{\mathscr{C}}\left(A \nabla_{\mathscr{C}}\right)$ on the open hypersurface $\mathscr{C}$ with the boundary $\partial \mathscr{C}:=\Gamma$, the following Green formulae are valid:

$$
\begin{aligned}
\left(\operatorname{div}_{\mathscr{S}}\right. & \left.\left(A \nabla_{\mathscr{C}} \varphi\right), \psi\right)_{\mathscr{C}} \\
= & \left(\left\langle\nu_{\Gamma},\left(A \nabla_{\mathscr{C}} \varphi\right)^{+}\right\rangle, \psi^{+}\right)_{\Gamma}-\left(A \nabla_{\mathscr{C}} \varphi, \nabla_{\mathscr{C}} \psi\right)_{\mathscr{C}}, \\
\left(\operatorname{div}_{\mathscr{S}}\right. & \left.\left(A \nabla_{\mathscr{C}} \varphi\right), \psi\right)_{\mathscr{C}}-\left(\varphi, \operatorname{div}_{\mathscr{S}}\left(A \nabla_{\mathscr{C}} \psi\right)\right)_{\mathscr{C}} \\
= & \left(\left\langle\nu_{\Gamma},\left(A \nabla_{\mathscr{C}} \varphi\right)^{+}\right\rangle, \psi^{+}\right)_{\Gamma}-\left(\varphi^{+},\left\langle\nu_{\Gamma},\left(A \nabla_{\mathscr{C}} \psi\right)^{+}\right\rangle\right)_{\Gamma},
\end{aligned}
$$

where $(\varphi, \psi)_{\mathscr{C}}$ denotes the scalar product of functions $\varphi, \psi \in$ $C^{\infty}(\mathscr{C})$. We have encountered already the normal boundary derivative $\left\langle\boldsymbol{\nu}_{\Gamma},\left(A \nabla_{\mathscr{C}} \varphi\right)^{+}\right\rangle$in the mixed $B V P(9)$.
Lemma 12 (see $[18,19]$ (Lax-Milgram)). Let $\mathfrak{B}$ be a Banach space and let $A(\varphi, \psi)$ be a continuous, bilinear form

$$
A(\cdot, \cdot): \mathfrak{B} \times \mathfrak{B} \longrightarrow \mathbb{R}
$$

and positive definite

$$
A(\varphi, \varphi) \geq C\|\varphi \mid \mathfrak{B}\|^{2} \quad \forall \varphi \in \mathfrak{B}, C>0 .
$$

Let $L(\cdot): \mathfrak{B} \rightarrow \mathbb{R}$ be a continuous linear functional. A linear equation

$$
A(\varphi, \psi)=L(\psi)
$$

has a unique solution $\varphi \in \mathfrak{B}$ for arbitrary $\psi \in \mathfrak{B}$.

\section{Mixed BVP for the Laplace-Beltrami Equation}

Let again $\mathscr{C} \subset \mathcal{S}$ be a smooth subsurface of a closed hypersurface $\mathcal{S}$ and let $\Gamma=\partial \mathscr{C} \neq \emptyset$ be its smooth boundary $\partial \mathscr{C}=\Gamma$. Let $\operatorname{div}_{\mathscr{C}} A \nabla_{\mathscr{C}}$ be the operator restricted to the hypersurface $\mathscr{C}$. The boundary is divided into two parts $\partial \mathscr{C}=\Gamma=\Gamma_{D} \cup \Gamma_{N}$ and in the present section we consider the boundary value problems for (8) with mixed boundary conditions in a weak classical formulation (4).

Note that functions $\varphi \in \mathbb{W}_{p}^{s}(\mathscr{C})$ and $\varphi \in \mathbb{M}_{p}^{s}(\mathscr{C})$ have the trace $\varphi^{+} \in \mathbb{W}_{p}^{s-1 / p}(\Gamma)$ on the boundary, provided $1<p<\infty$ and $s>1 / p$ (see [12] for details). Therefore if we look for a solution of the BVP (8) in the space $\mathbb{W}^{1}(\mathscr{C})$, the trace $u^{+}$on $\Gamma_{D}$ exists and belongs to the space $\mathbb{H}^{1 / 2}\left(\Gamma_{D}\right)$.

Concerning the existence of the Neumann trace $\left\langle\nu_{\Gamma}, A \nabla_{\mathscr{C}}\right\rangle^{+}$in (8) for a solution $u \in \mathbb{W}^{1}(\mathscr{C})$ is not guaranteed by the general trace theorem. But in this case, the first Green formula (56) ensures the existence of the Neumann trace. Indeed, by setting $\varphi=u$ and inserting the data $\left(\operatorname{div}_{\mathscr{C}} A \nabla_{\mathscr{C}}\right) u(t)=f(t)$ from $(8)$ into the first Green formula (56), we get the following:

$$
\begin{aligned}
\left(\left\langle\nu_{\Gamma},\right.\right. & \left.\left.\left(A \nabla_{\mathscr{C}} u\right)^{+}\right\rangle, \psi^{+}\right)_{\Gamma} \\
& -\left(A \nabla_{\mathscr{C}} u, \nabla_{\mathscr{C}} \psi\right)_{\mathscr{C}}=\left(\operatorname{div}_{\mathscr{C}}\left(A \nabla_{\mathscr{C}} u\right), \psi\right)_{\mathscr{C}}=(f, \psi)_{\mathscr{C}}
\end{aligned}
$$

and finally we get

$$
\left(\left\langle\boldsymbol{v}_{\Gamma},\left(A \nabla_{\mathscr{C}} u\right)^{+}\right\rangle, \psi^{+}\right)_{\Gamma}=(f, \psi)_{\mathscr{C}}+\left(A \nabla_{\mathscr{C}} u, \nabla_{\mathscr{C}} \psi\right)_{\mathscr{C}}
$$

for arbitrary $\psi \in \mathbb{W}^{1}(\mathscr{C})$. Since $\psi^{+} \in \mathbb{M}^{1 / 2}(\Gamma)$, the scalar product $\left(A \nabla_{\mathscr{C}} u, \nabla_{\mathscr{C}} \psi\right)_{\mathscr{C}}$ in the right-hand side of equality (61) is correctly defined and defines correct duality in the lefthand side of the equality. Since $\psi^{+} \in \mathbb{M}^{1 / 2}(\Gamma)$ is arbitrary, by the duality argument, this gives that $\left\langle\nu_{\Gamma},\left(A \nabla_{\mathscr{C}} u\right)^{+}\right\rangle$should be in the dual space, that is, in $\mathbb{H}^{-1 / 2}(\Gamma)$.

To prove the forthcoming theorem about the unique solvability of the BVP (61) we need more properties of trace operators (called retractions) and their inverses, called coretractions (see [12, Section 2.7]).

To keep the exposition simpler we recall a very particular case of Lemma 4.8 from [1], which we apply in the present investigation. 
Lemma 13 (see Lemma 4.8 in [1]). Let $s>0, s \notin \mathbb{N}, 1<$ $p<\infty, \mathbf{B}(D)$ be a normal differential operator of the first order defined in the vicinity of the boundary $\Gamma=\partial \operatorname{rtial} \mathscr{C}$ and let $\mathbf{A}(D)$ be a normal differential operator of the second order defined on the surface $\mathscr{C}$. Then there exists a continuous linear operator

$$
\mathscr{B}: \mathbb{W}_{p}^{s}(\Gamma) \otimes \mathbb{W}_{p}^{s-1}(\Gamma) \longrightarrow \mathbb{T}_{p}^{s+1 / p}(\mathscr{C})
$$

such that

$$
\begin{gathered}
(\mathscr{B} \Phi)^{+}=\varphi_{0}, \quad(\mathbf{B}(D) \mathscr{B} \Phi)^{+}=\varphi_{1}, \\
\mathbf{A}(D) \mathscr{B} \Phi \in \widetilde{\mathbb{A}}_{p}^{s-2+1 / p}(\mathscr{C}),
\end{gathered}
$$

for arbitrary pair of functions $\Phi=\left(\varphi_{0}, \varphi_{1}\right)^{\top}$, where $\varphi_{0} \in$ $\mathbb{W}_{p}^{s}(\Gamma)$ and $\varphi_{1} \in \mathbb{W}_{p}^{s-1}(\Gamma)$.

Theorem 14. The mixed boundary value problem (8) in the weak classical setting (4) and the additional constraint $f \in$ $\widetilde{\mathbb{A}}_{0}^{-1}(\Omega) \subset \widetilde{\mathbb{M}}^{-1}(\Omega)$ (see Remark 2 ), has a unique solution in the space $\mathbb{W}^{1}(\mathscr{C})$.

Proof. We commence by reduction of the BVP (8) to an equivalent one with the homogeneous Dirichlet condition. For this we extend the boundary data $g \in \mathbb{W}^{1 / 2}\left(\Gamma_{D}\right)$ up to some function $\widetilde{g} \in \mathbb{W}^{1 / 2}(\Gamma)$ on the entire boundary $\Gamma$ and apply Lemma 13: there exists a function $G \in \mathbb{W}^{1}(\mathscr{C})$ such that $G^{+}(t)=g(t)$ for $t \in \Gamma_{D}$ (actually $G^{+}=\widetilde{g}$ almost everywhere on the boundary) and $\operatorname{div}_{\mathscr{C}}\left(A \nabla_{\mathscr{C}} G\right) \in \widetilde{\mathbb{W}}^{-1}(\overline{\mathscr{C}})$.

For a new unknown function $v:=u-G$, we have the following equivalent reformulation of the BVP (8):

$$
\begin{gathered}
\operatorname{div}_{\mathscr{C}}\left(A \nabla_{\mathscr{C}} v\right)(t)=f_{0}(t), \quad t \in \mathscr{C}, \\
v^{+}(s)=0, \quad \text { on } \Gamma_{D}, \\
\left\langle v_{\Gamma}(s),\left(A \nabla_{\mathscr{C}} v\right)^{+}(s)\right\rangle=h_{0}(s), \quad \text { on } \Gamma_{N},
\end{gathered}
$$

where

$$
\begin{aligned}
& f_{0}:=f+ \operatorname{div}_{\mathscr{C}}\left(A \nabla_{\mathscr{C}} G\right) \in \widetilde{\mathbb{W}}^{-1}(\mathscr{C}), \\
& h_{0}:=h+\left\langle\nu_{\Gamma},\left(A \nabla_{\mathscr{C}} G\right)^{+}\right\rangle \in \mathbb{W}^{-1 / 2}\left(\Gamma_{N}\right), \\
& v^{+} \in \widetilde{\mathbb{W}}^{1 / 2}\left(\Gamma_{N}\right) .
\end{aligned}
$$

To justify the last inclusion $v^{+} \in \widetilde{\mathbb{W}}^{1 / 2}\left(\Gamma_{N}\right)$, note that, due to our construction, the trace of a solution on $\Gamma_{D}$ vanishes $\left.v^{+}\right|_{\Gamma_{D}}=0$.

Let $\Gamma_{0} \subset \Gamma$ be a part of the boundary and, by $\mathbb{W}^{\mathcal{s}}\left(\Gamma_{0}, \mathscr{C}\right)$, $s>1 / 2$, denote the space of functions $\varphi \in \mathbb{W}^{s}\left(\Gamma_{0}, \mathscr{C}\right)$ which has the trace on the boundary supported in $\Gamma_{0}$; that is, $\varphi^{+} \epsilon$ $\widetilde{\mathbb{W}}^{s-1 / 2}\left(\Gamma_{0}\right)$.
By inserting the data from the reformulated boundary value problem (64) into the first Green identity (56), where $\varphi=\psi=v$, we get

$$
\begin{aligned}
&\left(A \nabla_{\mathscr{C}} v, \nabla_{\mathscr{C}} v\right)_{\mathscr{C}} \\
&=\left(\left\langle v_{\Gamma},\left(A \nabla_{\mathscr{C}} v\right)^{+}\right\rangle, v^{+}\right)_{\Gamma_{D}}+\left(\left\langle v_{\Gamma},\left(A \nabla_{\mathscr{C}} v\right)^{+}\right\rangle, v^{+}\right)_{\Gamma_{N}} \\
&-\left(\operatorname{div}_{\mathscr{C}}\left(A \nabla_{\mathscr{C}} v\right), v\right)_{\mathscr{C}}=\left(h_{0}, v^{+}\right)_{\Gamma_{N}}-\left(f_{0}, v\right)_{\mathscr{C}}, \\
& v \in \widetilde{\mathbb{W}}^{1}\left(\Gamma_{N}, \mathscr{C}\right) .
\end{aligned}
$$

In the left-hand side of the equality (66) we have a symmetric bilinear form, which is positive definite

$$
\left(A \nabla_{\mathscr{C}} v, \nabla_{\mathscr{C}} v\right)_{\mathscr{C}} \geqslant C\left\|\nabla_{\mathscr{C}} v\right\|^{2} \quad \forall v \in \widetilde{\mathbb{W}}^{1}\left(\Gamma_{N}, \mathscr{C}\right),
$$

because $A=\left[a_{j k}\right]_{n \times n}$ is strictly positive definite matrix. On the other hand, all functions on the space $\widetilde{\mathbb{W}}^{1}\left(\Gamma_{N}, \mathscr{C}\right)$ vanish on the part of the boundary $\Gamma_{D}$, and $\nabla_{\mathscr{C}} v$ defines an equivalent norm on this space

$$
\left\|\nabla_{\mathscr{C}} v\right\| \leqslant\left\|v \mid \mathbb{W}^{1}\left(\Gamma_{N}\right)\right\| \leqslant C_{1}\left\|\nabla_{\mathscr{C}} v\right\| \quad \forall v \in \widetilde{\mathbb{W}}^{1}\left(\Gamma_{N}, \mathscr{C}\right) .
$$

From (67) and (68) follows the positive definiteness:

$$
\left(A \nabla_{\mathscr{C}} v, \nabla_{\mathscr{C}} v\right)_{\mathscr{C}} \geqslant C_{2}\left\|v \mid \mathbb{W}^{1}\left(\Gamma_{N}\right)\right\|^{2} \quad \forall v \in \widetilde{\mathbb{W}}^{1}\left(\Gamma_{N}, \mathscr{C}\right) .
$$

$\left(h_{0}, v^{+}\right)_{\Gamma_{N}}$ and $\left(f_{0}, v\right)_{\mathscr{C}}$ from equality (66) are correctly defined continuous functionals, because $h_{0} \in \mathbb{W}^{-1 / 2}\left(\Gamma_{N}\right), f_{0} \in \widetilde{\mathbb{W}}^{-1}(\mathscr{C})$, while their counterparts in the functional belong to the dual spaces $v^{+} \in \widetilde{\mathbb{W}}^{1 / 2}\left(\Gamma_{N}\right)$ and $v \in \widetilde{\mathbb{W}}^{1}\left(\Gamma_{N}, \mathscr{C}\right) \subset \mathbb{W}^{1}(\mathscr{C})$.

The Lax-Milgram Lemma 12 accomplishes the proof.

Remark 15. The Lax-Milgram Lemma 12 can be applied to the Dirichlet BVP (1), (4), but only after equivalent reformulation: due to Lemma 13 we can pick up a function $G \in \mathbb{W}^{1}(\mathscr{C})$ such that $G^{+}=g$ and $\operatorname{div}_{\mathscr{C}}\left(A \nabla_{\mathscr{C}} G\right) \in \widetilde{\mathbb{W}}^{-1}(\mathscr{C})$.

For a new unknown function $v:=u-G$, we have the following equivalent reformulation of the BVP (5):

$$
\begin{gathered}
\operatorname{div}_{\mathscr{C}}\left(A \nabla_{\mathscr{C}} v\right)(t)=f_{0}(t), \quad t \in \mathscr{C}, \\
v^{+}(s)=0, \quad \text { on } \Gamma,
\end{gathered}
$$

where

$$
f_{0}:=f+\operatorname{div}_{\mathscr{C}}\left(A \nabla_{\mathscr{C}} G\right) \in \widetilde{\mathbb{W}}^{-1}(\mathscr{C}), \quad v \in \widetilde{\mathbb{W}}^{1}(\mathscr{C}) .
$$

By inserting the data from the reformulated boundary value problem (70) into the first Green identity (56), where $\varphi=\psi=$ $v$, we get

$$
\begin{aligned}
\left(A \nabla_{\mathscr{C}} v, \nabla_{\mathscr{C}} v\right)_{\mathscr{C}}= & \left(\left\langle\nu_{\Gamma},\left(A \nabla_{\mathscr{C}} v\right)^{+}\right\rangle, v^{+}\right)_{\Gamma} \\
& -\left(\operatorname{div}_{\mathscr{C}}\left(A \nabla_{\mathscr{C}} v\right), v\right)_{\mathscr{C}}=-\left(f_{0}, v\right)_{\mathscr{C}}, \\
v & \in \widetilde{\mathbb{W}}^{1}(\mathscr{C}) .
\end{aligned}
$$

Now the Lax-Milgram Lemma can be applied, which proves the unique solvability of the Dirichlet BVP (1), (4). 
Remark 16. The Lax-Milgram Lemma 12 can be applied to the Neumann BVP (2), (4):

$$
\begin{gathered}
\operatorname{div}_{\mathscr{C}}\left(A \nabla_{\mathscr{C}} v\right)(t)=f_{0}(t), \quad t \in \mathscr{C}, \\
\left\langle\nu_{\Gamma},\left(A \nabla_{\mathscr{C}} v\right)^{+}\right\rangle(s)=0, \quad \text { on } \Gamma .
\end{gathered}
$$

By inserting the data from the boundary value problem (2) into the first Green identity (56), where $\varphi=\psi=u$, we get

$$
\begin{aligned}
\left(A \nabla_{\mathscr{C}} u, \nabla_{\mathscr{C}} u\right)_{\mathscr{C}} & \\
\quad & =\left(\left\langle\nu_{\Gamma}, A \nabla_{\mathscr{C}} u\right\rangle^{+}, u^{+}\right)_{\Gamma}-\left(\left(\operatorname{div}_{\mathscr{C}} A \nabla_{\mathscr{C}}\right) u, u\right)_{\mathscr{C}} \\
& =(h, u)_{\Gamma}-(f, u)_{\mathscr{C}}, \quad u \in \mathbb{W}_{2, \#}^{1}(\mathscr{C}) .
\end{aligned}
$$

We need the compatibility condition (6) to ensure that the equality in (74) will be fulfilled on the subspace $\mathbb{W}_{2, \#}^{1}(\mathscr{C})$ : if $v=$ const, the left-hand side vanishes, while the right-hand side vanishes, $(h, \text { const })_{\Gamma}-(f \text {, const })_{\mathscr{C}}=0$, if and only if the compatibility condition (6) holds:

$$
(h, 1)_{\Gamma}-(f, 1)_{\mathscr{C}}=\oint_{\Gamma} h(s) d s-\int_{\mathscr{C}} f(y) d \sigma=0,
$$

and the last representation in the equality is possible if $h$ and $f$ are regular integrable functions.

Due to (55), the bilinear form $\left(A \nabla_{\mathscr{C}} v, \nabla_{\mathscr{C}} v\right)_{\mathscr{C}}$ is positive definite and both functionals in the right-hand side of (74) are bounded on the subspace $\mathbb{W}_{2, \#}^{1}(\mathscr{C})$. Now we can apply the Lax-Milgram Lemma and prove the existence of a unique solution $v \in \mathbb{W}_{2, \#}^{1}(\mathscr{C})$ to the Neumann BVP (2), (4), provided the compatibility9 condition (6) holds.

\section{Conflict of Interests}

The authors declare that there is no conflict of interests regarding the publication of this paper.

\section{Acknowledgments}

The first and the second authors were supported by the Grant of the Shota Rustaveli Georgian National Science Foundation GNSF/DI/10/5-101/12 and the second author was also supported by the Grant of the Shota Rustaveli Georgian National Science Foundation GNSF/PG/76/5-101/13.

\section{References}

[1] R. Duduchava, "The Green formula and layer potentials," Integral Equations and Operator Theory, vol. 41, no. 2, pp. 127178, 2001.

[2] R. Duduchava, D. Mitrea, and M. Mitrea, "Differential operators and boundary value problems on hypersurfaces," Mathematische Nachrichten, vol. 279, no. 9-10, pp. 996-1023, 2006.

[3] R. Duduchava, "Partial differential equations on hypersurfaces," Georgian Academy of Sciences A: Memoirs on Differential Equations and Mathematical Physics, vol. 48, pp. 19-74, 2009.
[4] M. Mitrea and M. Taylor, "Potential theory on Lipschitz domains in Riemannian manifolds: Sobolev-Besov space results and the Poisson problem," Journal of Functional Analysis, vol. 176, no. 1, pp. 1-79, 2000.

[5] H. Le Dret, Numerical Approximation of PDEs, Class Notes M1 Mathematics, 2011-2012, 2011, http://www.ann.jussieu.fr/ledret/ M1ApproxPDE.html.

[6] W. Haack, Elementare Differentialgeometrie (Lehrbücher und Monographien aus dem Gebiete der Exakten Wissenschaften), Birkhäauser, Basel, Switzerland, 1955, (German).

[7] R. Aris, Vectors, Tensors, and the Basic Equations of Fluid Mechanics, Prentice-Hall, Englewood Cliffs, NJ, USA, 1962.

[8] P. G. Ciarlet, Introduction to Linear Shell Theory, vol. 1 of Series in Applied Mathematics, Gauthier-Villars, Paris, France, 1998.

[9] L. Andersson and P. T. Chruściel, "Hyperboloidal Cauchy data for vacuum Einstein equations and obstructions to smoothness of null infinity," Physical Review Letters, vol. 70, no. 19, pp. 28292832, 1993.

[10] R. Temam and M. Ziane, "Navier-stokes equations in thin spherical domains," in Optimization Methods in Partial Differential Equations, vol. 209 of Contemporaty Math., pp. 281-314, AMS, 1996.

[11] R. Duduchava, "A revised asymptotic model of a shell," Memoirs on Differential Equations and Mathematical Physics, vol. 52, pp. 65-108, 2011.

[12] H. Triebel, Interpolation Theory, Function Spaces, Differential Operators, Johann Ambrosius Barth, Heidelberg, Germany, 2nd edition, 1995.

[13] L. Hörmander, The Analysis of Linear Partial Differential Operators IV, Springer, Heidelberg, Germany, 1983.

[14] G. C. Hsiao and W. L. Wendland, Boundary Integral Equations, vol. 164 of Applied Mathematical Sciences Series, Springer, Berlin, Germany, 2008.

[15] M. Shubin, Pseudodifferential Operators and Spectral Theory, Springer, Berlin, Germany, 1987.

[16] M. E. Taylor, Pseudodifferential Operators, Princeton University Press, Princeton, NJ, USA, 1981.

[17] R. Duduchava, "On Noether theorems for singular integral equations," in Proceedings of the Symposium on Mechanics and Related Problems of Analysis, vol. 1, pp. 19-52, Metsniereba, Tbilisi, Georgia, 1973, (Russian).

[18] P. D. Lax and A. N. Milgram, "Parabolic equations," in Contributions to the theory of partial differential equations, Annals of Mathematics Studies, pp. 167-190, Princet on University Press, Princeton, NJ, USA, 1954.

[19] J. Lions and E. Magenes, Non-Homogeneous Boundary Value Problems and Applications. Vol. I, Springer, Heidelberg, Germany, 1972. 


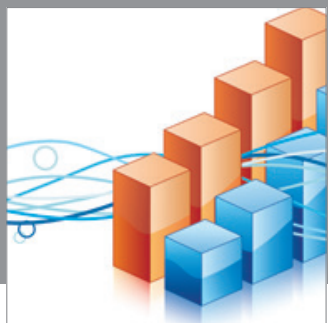

Advances in

Operations Research

mansans

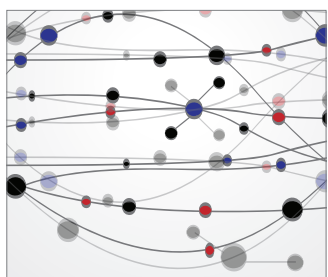

The Scientific World Journal
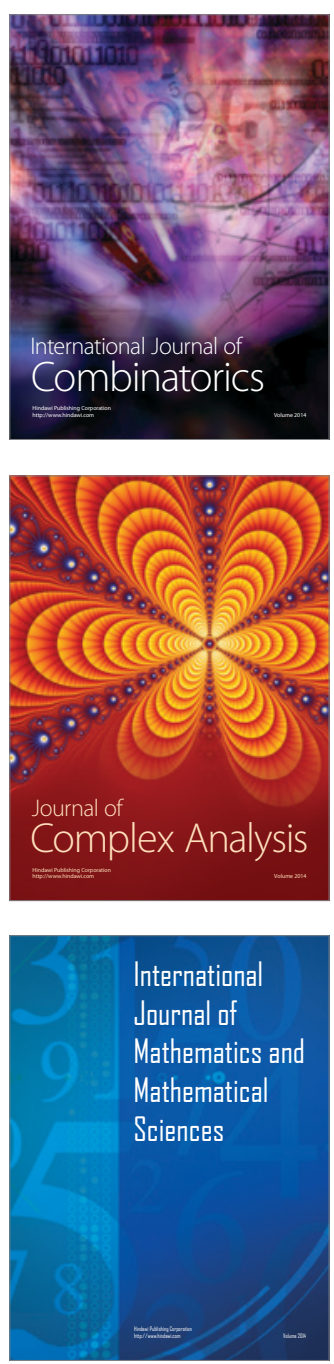
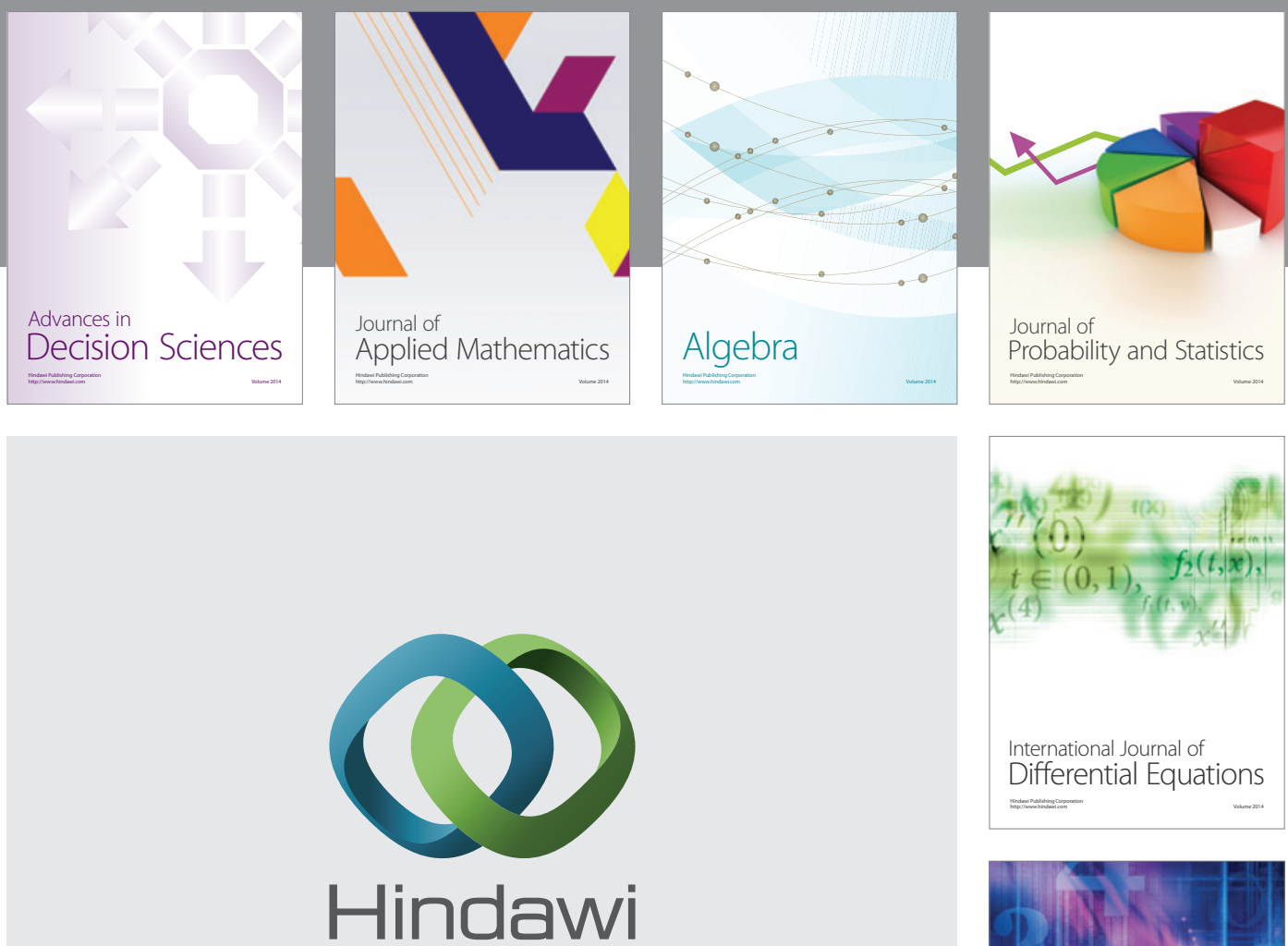

Submit your manuscripts at http://www.hindawi.com
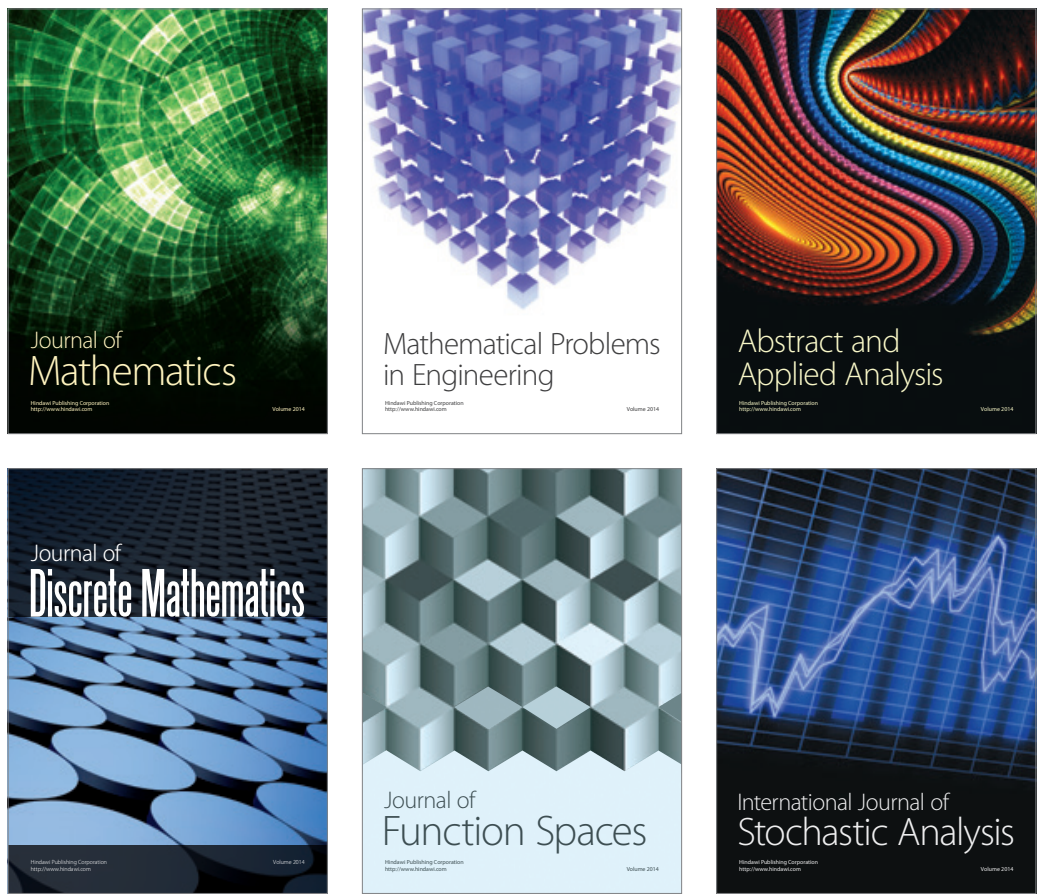

Journal of

Function Spaces

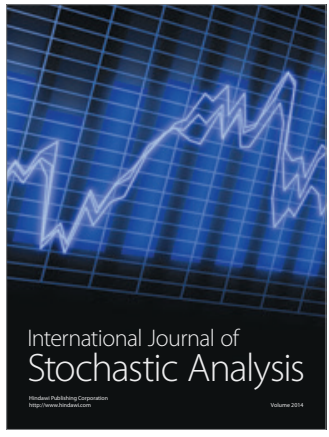

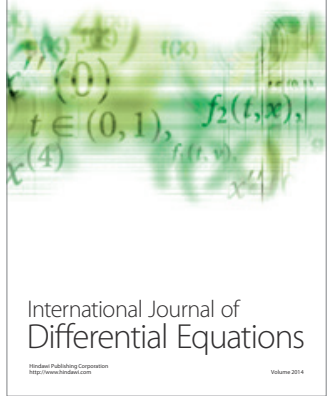
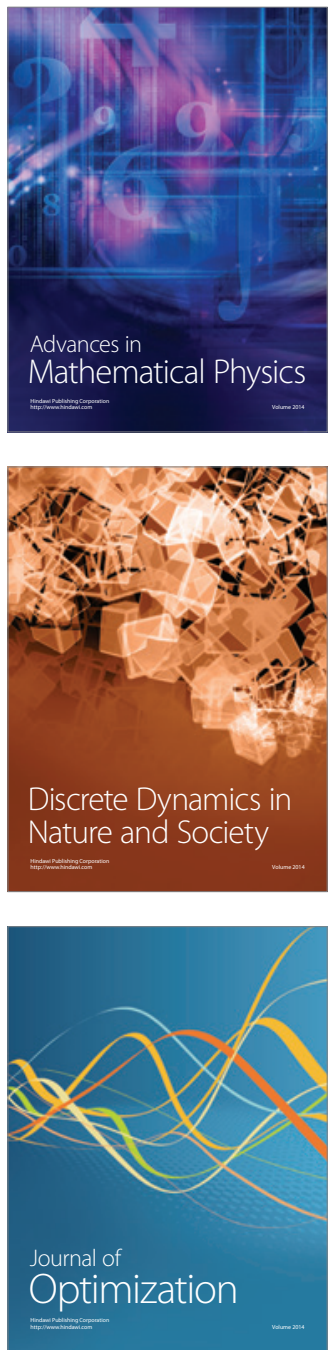